\title{
Understanding Initial Formation Stages of Nanomaterials Using Cryo-TEM
}

\author{
Katherine A. Spoth ${ }^{1}$, Kai Ma ${ }^{2}$, Ulrich Wiesner ${ }^{2}$ and Lena F. Kourkoutis ${ }^{1,3}$
}

1. School of Applied and Engineering Physics, Cornell University, Ithaca, New York, USA.

2. Dept. of Materials Science and Engineering, Cornell University, Ithaca, New York, USA.

3. Kavli Institute at Cornell for Nanoscale Science, Ithaca, New York, USA.

Controlled solution-based synthesis offers a path towards nanomaterials with designed structure and functionalities. For instance, amphiphilic block copolymers [1] and surfactant molecules which self-assemble in solution are used as templates for the production of tailored nanostructures. The initial steps in these formation processes are often critical. Cryo-TEM allows the direct observation of specimens in solution and therefore is a valuable tool for characterizing solutionbased formation processes of nanomaterials [2]. Here, we use cryo-TEM to study the formation process of hybrid organic-inorganic silica nanoparticles, $10 \mathrm{~nm}$ in diameter, formed by the condensation of silica onto organic micelles. We have captured steps in the synthesis of these particles by snap-freezing the structures in solution upon the addition of each component, revealing the evolution from isolated micelles to aligned silica rings.

Porous silica nanomaterials show promise for applications in fields from energy generation and catalysis to nanomedicine. For example, one study of single-pore silica nanoparticles demonstrates their potential for drug delivery applications - the pore allows material to be encapsulated with the particles small enough to be removed by the kidneys, avoiding toxic effects [3]. While porous silica materials have been extensively studied, their formation is not well understood. Furthermore, they are overwhelmingly characterized by conventional TEM in which the drying process can alter the materials' structure. Cryo-TEM allows direct observation of the solution-based formation process without drying artifacts. Revealing the materials' formation pathways is vital to controlling their synthesis so that new materials can be designed.

Steps in the formation process of 10-nm single-pore ring particles are shown in Figure 1, starting with spherical micelles in solution (Figure 1a) consisting of a hydrophobic pore-expanding material surrounded by surfactant molecules. Individual ring particles, shown in Figure 1b, form as primary silica particles which nucleate in solution attach to the micelles and align to form a ring. The details of the alignment process are not clearly understood at this point. Figure 1c shows the result of adding hydrophilic polymer which associates with silica and further promotes the formation of uniform chains of rings in solution, which in turn assemble into 3D helical structures.

Another route to ordering is by condensation of additional silica monomers onto the individual ring particles. Figure 2 a shows the ring particles formed by attachment of primary particles to the two-component micelles. The ring size is then increased by condensation of silica monomers onto existing rings. As additional precursor is dosed, individual rings network into chain-like structures (Figure 2b-d) and the diameter of individual rings increases. Images of the dry sample (Figure 2eh) show that the drying process causes a dramatic increase in particle density on the grid, obscuring the structural differences that are easily observed in the cryo-immobilized sample. Cryo-TEM reveals the solution-dependent structure without potential structural changes from drying processes, allowing us to capture the initial stages of formation in hybrid silica/organic nanostructures [4]. 
References:

[1] H. Cui et al., Science 317, 647 (2007).

[2] C. C. M. C. Carcouët et al, Nano Letters 14, 1433 (2014).

[3] K. Ma et al, Chemistry of Materials 27, 4119 (2015).

[4] This work was supported by the Cornell Center for Materials Research with funding from the NSF MRSEC program (DMR-1120296).
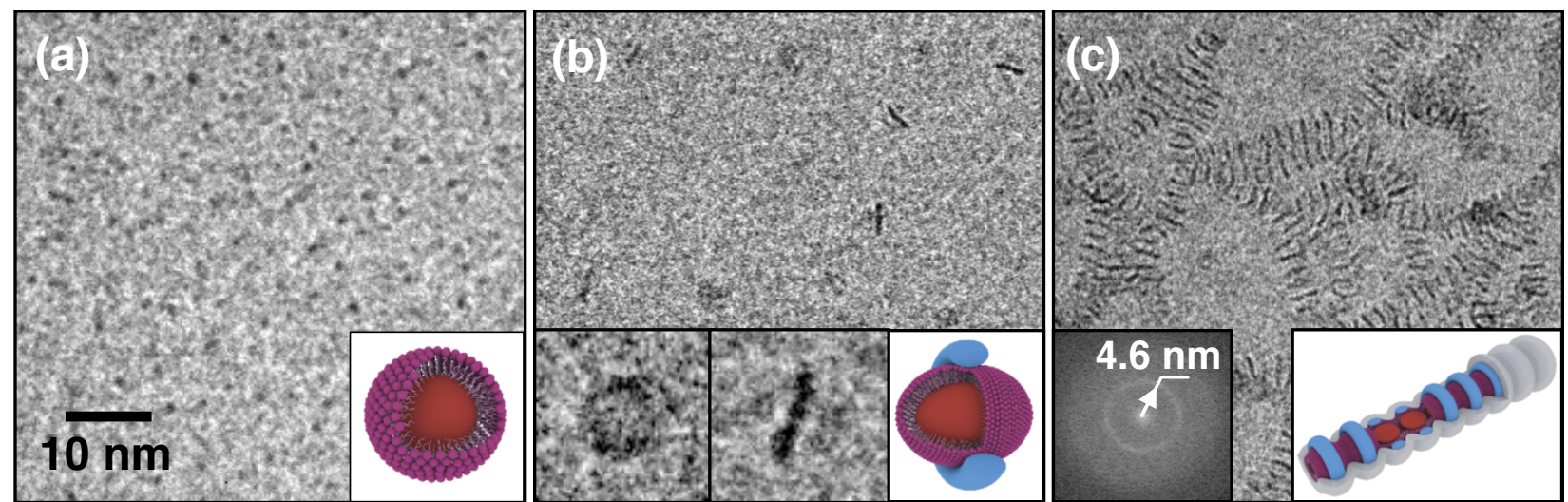

Figure 1. Formation process of silica nanoparticles in solution imaged by cryo-TEM. Individual micelles consist of pore expander material plus surfactant. (a). Silica precursor is added to solution and rings form (b), with individual rings magnified in inset (field of view $18 \mathrm{~nm}$ ). Uniform chains form upon addition of hydrophilic polymer (c) with average spacing of $\sim 4.6 \mathrm{~nm}$.

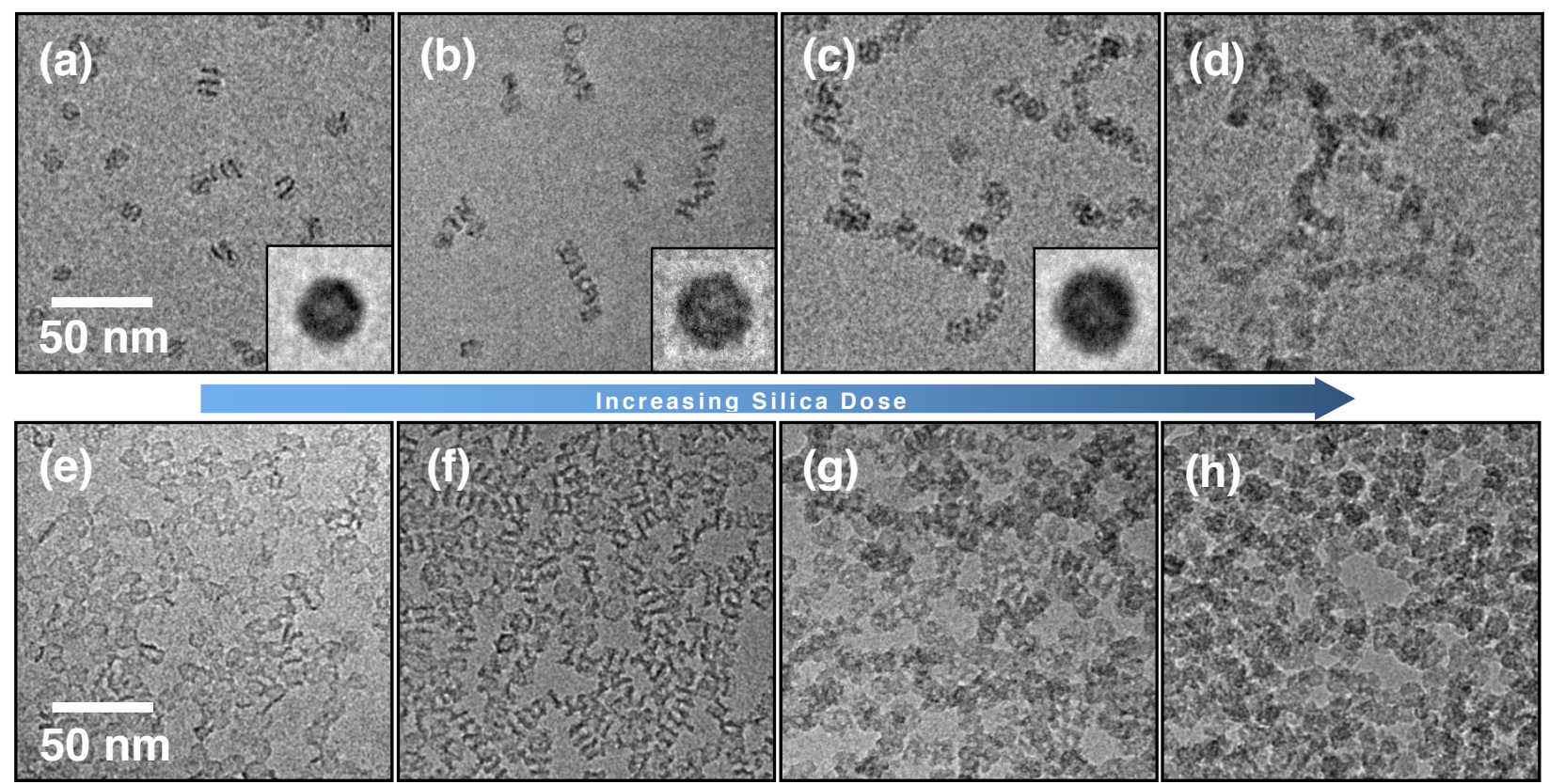

Figure 2. TEM images of nanoparticles dosed with additional silica showing increased particle diameter and particle order. Cryo-TEM images (a-d) reveal the lengthening of chains with irregular particle spacing as additional silica is added. Insets (a-c, field of view $20 \mathrm{~nm}$ ) depict averages of 25 or more particles from each condition and reveal a diameter increase from 10 to $13 \mathrm{~nm}$. Conventional TEM images of the samples when dried indicate linking particles, but without cryoTEM it is unclear to what extent this ordering is caused by the drying process (e-h). 